\title{
Mutation Screening of Elongation Factor 2 in Shwachman-Diamond Syndrome Patients Lacking Mutations in the SBDS Gene
}

\author{
Elena Nicolis ${ }^{1}$ and Marco Cipolli ${ }^{2}$ \\ ${ }^{1}$ Laboratory of Molecular Pathology, University Hospital of Verona, Piazzale Stefani, 37126 Verona, Italy \\ ${ }^{2}$ Cystic Fibrosis Center, University Hospital of Verona, Piazzale Stefani, 37126 Verona, Italy \\ Correspondence should be addressed to Elena Nicolis; elenalbius@gmail.com
}

Received 10 October 2012; Accepted 27 October 2012

Academic Editors: B. Blaumeiser, B.-H. Jeong, J. Moreaux, and M. Romkes

Copyright (C) 2013 E. Nicolis and M. Cipolli. This is an open access article distributed under the Creative Commons Attribution License, which permits unrestricted use, distribution, and reproduction in any medium, provided the original work is properly cited.

\begin{abstract}
Shwachman-Diamond syndrome is an autosomal recessive disorder characterized by bone marrow failure, pancreatic insufficiency, and skeletal abnormalities. Mutations in SBDS gene explain, by literature, $90 \%$ of SDS cases. The Italian experience shows that only the $5 \%$ of individuals diagnosed as affected by SDS on clinical and hematological grounds lack mutations in the SBDS gene. It is well established that SBDS protein is essential for the assembly of mature ribosomes. The yeast SBDS ortholog functions within a pathway containing elongation factor-like 1, homologous to human GTPase elongation factor-2, to promote the release and recycling of the nucleolar shuttling factor Tif6 from cytoplasmic pre-60S subunits in a cascade targeted to form the active ribosome. We considered that mutations of genes that disrupt pathways shared by SBDS may result in disease with comparable clinical features. EEF2 was evaluated as a candidate gene by mutation screening in clinically defined SDS which lack mutations in the SBDS gene. To date, no deleterious mutations were found in EEF2 in four Italian patients without SBDS mutations, but with a clinical diagnosis of SDS.
\end{abstract}

\section{Introduction}

Shwachman-Diamond-syndrome- (SDS-)associated mutations were described in a gene-designed ShwachmanBodian-Diamond syndrome (SBDS) [1] that encodes a member of a highly conserved protein family, with orthologues in diverse species including archaea, plants, and eukaryotes. Structural studies of the Archaeoglobus fulgidus SBDS ortholog [2] revealed the presence of three domains. The $\mathrm{N}$-terminal domain is identical to the single domain yeast protein Yhr087wp that is implicated in RNA metabolism. The protein that is most closely structurally related to the second domain is the C-terminal domain of E. coli, RuvA, that is involved in Holliday junction recognition during the recombination event. The closest structural homologue to C-terminal third domain of Archaeoglobus fulgidus SBDS ortholog is the fifth domain of yeast S. cerevisiae elongation factor 2 [2]. The SBDS mRNA is widely expressed throughout the human tissue [1]; furthermore, immunofluorecence studies showed that the SBDS protein is localized to both the nucleus and the cytoplasm, but is particularly concentrated within the nucleolus [3]. The nucleolus is best known as a site of ribosome biogenesis. An interesting discovery from proteomic studies of the nucleolus [4-6] was that approximately $30 \%$ of nucleolar proteins constituted either novel or uncharacterized proteins. It is known that RNA processing factors are localized in the nucleolus, which is intriguing since the SBDS protein has been postulated to play a role in RNA processing based on data from SBDS orthologues. Consistent with an essential cellular function, loss of the mouse SBDS ortholog results in early embryonic lethality [7]. Menne et al. [8] demonstrated a critical function for the yeast SBDS ortholog, called Sdo1, in late maturation of cytoplasmic pre-60S ribosomes. In the eukaryotic cells $60 \mathrm{~S}$, is one of the two subunits of the polyribosome $80 \mathrm{~S}$, the important "machinery" involved in the translation from mRNA to protein. Menne and colleagues showed that Sdo1 functions within a pathway containing elongation factor-like 
TABLE 1: Primers sequences and amplicon size of the 11 fragments used for the analysis of the EEF2 gene.

\begin{tabular}{lccc}
\hline Exon & Forward primer & Reverse primer & Amplicon length \\
\hline 1 & cctataaaaagctgagtgttgacgtc & caccagcgaggcagggt & 142 \\
2 & gtgccatgctgtgttcctggaaat & tcgctggactgaacctcactcatt & 425 \\
$3-4$ & atgaatcttgggaccaagtcggtg & ctgtcacccaacattcctggcaaa & 872 \\
$5-6$ & gtctactgaagaaagcctgcgtct & tccagatcttaagagaggagccctga & 765 \\
7 & tgggctttcttgtttcttggtggg & tgtcagagcatccggaaacagca & 211 \\
$8-9$ & tggcttcaagagaggaagcgtgta & atgctccttacttctagctccca & 649 \\
10 & tgaagaggatgtttcctgacagcc & agtctccatttacagccacagcca & 582 \\
11 & ttcccagcaccttcctgaaatct & cacatgcctgtggaagactgcaaa & 396 \\
12 & aggatgcgtctgtgtgtaaggtca & atgaggtccctctagagcctggaa & \\
$13-14$ & acttggcaggtggagggcaa & tcaggacgcctcctttaacacctt & \\
15 & tgagctcctgacaggactttccttctgccct & ccctgcgaagacaccgtgaaa & 563 \\
\hline
\end{tabular}

1 (Efl-1) to promote the release and recycling of the nucleolar shuttling factor Tif6 from cytoplasmic pre-60S subunits. 60S, free from Tif6, is able to assemble to subunit $40 \mathrm{~S}$ and to form the active ribosome. Efl-1 is a cytoplasmic GTPase that is highly homologous to human GTPase elongation factor2 (EEF2, formerly known as EF-2), and it promotes a GTPdependent dissociation of Tif6 from 60S ribosomes in vitro [9]. The structural homology between Efl1 and the ribosomal translocase EEF2 [9] indicates that they bind to a similar site at the GTPase center of the ribosome $[10,11]$. Structural homology between the archaeal SBDS protein and the fifth domain of EEF 2 [2] supports the hypothesis that Sdo1 may also bind in the vicinity of the GTPase center of the $60 \mathrm{~S}$ subunit. Recently, Sezgin et al. [12] demonstrated that the knockdown of SBDS leads to growth inhibition and defects in ribosome maturation, suggesting a role for wildtype SBDS in nuclear export of pre-60S subunits. It is known that gene products mutated in some inherited bone marrow failure syndromes such as dyskeratosis congenital and DiamondBlackfan anemia are all involved in different aspects of ribosome synthesis. Diamond-Blackfan anemia is associated with mutations in the RPS19 [13] and RPS24 [14] genes that encode structural components of the 40S ribosomal subunit. The human Cbf5 homolog dyskerin, DKC1, is mutated in the bone marrow failure disorder dyskeratosis congenita [15]. The early embryonic lethality associated with defective epiblast formation in SBDS-deficient mice, taken together with the high levels of SBDS expression in rapidly proliferating tissues [6], is entirely consistent with a critical requirement for SBDS in the de novo protein synthesis during the development. The SDS phenotype may reflect the sensitivity of specific cell types that are unable to dynamically upregulate ribosome synthesis at critical points during the development as a consequence of SBDS deficiency. Sdo1 is therefore a new component of a critical conserved pathway that is required for translational activation of ribosomes and links defective $60 \mathrm{~S}$ subunit maturation to an inherited bone marrow failure syndrome associate with leukaemia predisposition. It has been reported [1] that about $10 \%$ of individuals with SDS lack mutations in the SBDS gene. Thereby, EEF2 was evaluated as a candidate gene by mutation screening in clinically defined SDS patients who lack mutations in the SBDS gene.

\section{Methods and Materials}

EEF 2 gene is composed of $3164 \mathrm{bp}$, with a coding sequence of $2577 \mathrm{bp}$, divided into 15 exons. Screening has been performed in the coding and flanking region of a selected subgroup of SDS patients classified on rigorous clinical criteria [16] but were negative for SBDS mutations.

2.1. Patient Enrollment. The subgroup of SDS patients was ascertained based on an objective clinical evidence of bone marrow hypoplasia (neutropenia) and exocrine pancreatic dysfunction (low serum trypsinogen and isoamylase, abnormal $72 \mathrm{hr}$ fecal fat study, fatty pancreas) as well as confirmation of the absence of SBDS mutation on both alleles. Four over 80 Italian patients were selected (5\%).

2.2. DNA Extraction. Peripheral blood samples from the subgroup of SDS patients were collected (4-8 mL) by venipuncture in EDTA-vacutainer tubes. Genomic DNA was extracted from peripheral blood samples using the salting out method [17].

2.3. EEF 2 Sequencing Analysis. We characterized the genotype of the subgroup of SDS patients by direct sequencing. The primers used for the amplification and sequencing of the 15 exons and their flanking intron/exon junctions were chosen by the Primer Express Software (Applied Biosystems) (see Table 1).

PCRs were carried out in a $50 \mu \mathrm{l}$ reaction volume containing $350 \mathrm{ng}$ of genomic DNA, $0.5 \mu \mathrm{M}$ primers, $200 \mu \mathrm{M}$ dNTP, $5 \mu \mathrm{L} 10 \times$ PCR buffer, and 2U FastStart Taq DNA Polymerase (Roche, Mannheim, Germany). PCR products were checked by $2 \%$ agarose gel electrophoresis. The amplicons were then purified by a spin column purification method (GENOMED $\mathrm{GmbH}$, Löhne, Germany) and prepared for the sequencing analysis following the BMR Genomics Service instructions (BMR Genomics, Padua, Italy). 


\section{Results and Discussion}

SDS is a rare genetic syndrome: Minelli et al. [18] shows that the number of mutation proven SDS cases in Italy is $1 / 168,000$. Consequently, the carrier incidence expected in the Italian population is $1 / 205$. In the present study, we identified 4 out of 80 Italian patients, classified based on rigorous clinical criteria as having SDS but lacking mutations in the $S B D S$ gene. Our diagnosis criteria are well described in the guidelines by Dror et al. [16], and it allowed us to identify a small group of SDS-like patients (5\%), a smaller group, and then the one indicated by literature (10\%); as the clinical diagnosis of SDS is usually difficult to make due to the complexity of the clinical phenotype, rarity of the disease, and the paucity of large studies, we suppose that an overestimation of SDS diagnosis is the reason of such discrepancy. To date, no deleterious mutations were found in EEF2 in four Italian patients without SBDS mutations. The known SNP rs36525 in intron 8, with a reported MAF of 0.43 , was detected in one patient consistent with this polymorphism having no clinical significance. We point out that the present work aims to perform mutation screening of the coding and flanking regions of EEF2 gene; causing disaese variations might be present in noncoding regions of the EEF2 gene or be due to copy number variations. Why SDS-like patients with no SBDS mutations are observed could be due at least to 2 possibilities. It is possible that patients with normal SBDS alleles have been misclassified and actually have a distinct clinical syndrome. Alternatively, SDS may be a genetically heterogenous disorder. Mutations of a gene or genes that disrupt a pathway shared by SBDS may result in diseases with identical clinical features. We are now working on the screening of other genes with the same characteristics.

\section{Conflict of Interests}

The authors declare that they do not have any conflict of interests in the submitted paper; in particular, they do not have any direct financial relation with the commercial identity mentioned in their paper that might lead to a conflict of interests.

\section{Acknowledgments}

This work was funded by grants from AISS-Associazione Italiana Sindrome di Shwachman. The authors gratefully acknowledge. Professor Cesare Danesino and Dr. Antonella Minelli, Department of Molecular Medicine, University of Pavia, Fondazione IRCCS Policlinico San Matteo Pavia, Italy; Dr. Furio Poli, Cystic Fibrosis Center, IRCCS Burlo Garofolo, Trieste, Italy. Special thanks go to the patients and their families.

\section{References}

[1] G. R. B. Boocock, J. A. Morrison, M. Popovic et al., "Mutations in SBDS are associated with Shwachman-Diamond syndrome," Nature Genetics, vol. 33, no. 1, pp. 97-101, 2003.
[2] C. Shammas, T. F. Menne, C. Hilcenko et al., "Structural and mutational analysis of the SBDS protein family: insight into the leukemia-associated Shwachman-Diamond Syndrome," Journal of Biological Chemistry, vol. 280, no. 19, pp. 19221-19229, 2005.

[3] K. M. Austin, R. J. Leary, and A. Shimamura, "The ShwachmanDiamond SBDS protein localizes to the nucleolus," Blood, vol. 106, no. 4, pp. 1253-1258, 2005.

[4] J. S. Andersen, C. E. Lyon, A. H. Fox et al., "Directed proteomic analysis of the human nucleolus," Current Biology, vol. 12, no. 1, pp. 1-11, 2002.

[5] A. Scherl, Y. Couté, C. Déon et al., "Functional proteomic analysis of human nucleolus," Molecular Biology of the Cell, vol. 13, no. 11, pp. 4100-4109, 2002.

[6] A. K. L. Leung, J. S. Andersen, M. Mann, and A. I. Lamond, "Bioinformatic analysis of the nucleolus," Biochemical Journal, vol. 376, no. 3, pp. 553-569, 2003.

[7] S. Zhang, M. Shi, C. C. Hui, and J. M. Rommens, "Loss of the mouse ortholog of the Shwachman-Diamond syndrome gene (Sbds) results in early embryonic lethality," Molecular and Cellular Biology, vol. 26, no. 17, pp. 6656-6663, 2006.

[8] T. F. Menne, B. Goyenechea, N. Sánchez-Puig et al., “The Shwachman-Bodian-Diamond syndrome protein mediates translational activation of ribosomes in yeast," Nature Genetics, vol. 39, no. 4, pp. 486-495, 2007.

[9] B. Senger, D. L. J. Lafontaine, J. S. Graindorge et al., "The nucle(ol)ar Tif6p and Efl1p are required for a late cytoplasmic step of ribosome synthesis," Molecular Cell, vol. 8, no. 6, pp. 1363-1373, 2001.

[10] J. S. Graindorge, J. C. Rousselle, B. Senger et al., "Deletion of EFL1 results in heterogeneity of the $60 \mathrm{~S}$ GTPase-associated rRNA conformation," Journal of Molecular Biology, vol. 352, no. 2, pp. 355-369, 2005.

[11] C. M. T. Spahn, M. G. Gomez-Lorenzo, R. A. Grassucci et al., "Domain movements of elongation factor eEF2 and the eukaryotic $80 \mathrm{~S}$ ribosome facilitate tRNA translocation," The EMBO Journal, vol. 23, no. 5, pp. 1008-1019, 2004.

[12] G. Sezgin, A. L. Henson, A. Nihrane et al., "Impaired growth, hematopoieticcolony formation, and ribosome maturation in human cells depleted of Shwachman-Diamond syndrome protein SBDS," Pediatric Blood \& Cancer. In press.

[13] N. Draptchinskaia, P. Gustavsson, B. Andersson et al., "The gene encoding ribosomal protein S19 is mutated in DiamondBlackfan anaemia," Nature Genetics, vol. 21, no. 2, pp. 169-175, 1999.

[14] H. T. Gazda, A. Grabowska, L. B. Merida-Long et al., "Ribosomal protein S24 gene is mutated in Diamond-Blackfan anemia," American Journal of Human Genetics, vol. 79, no. 6, pp. 1110-1118, 2006.

[15] S. W. Knight, N. S. Heiss, T. J. Vulliamy et al., "X-linked dyskeratosis congenita is predominatly caused by missense mutations in the DKC1 gene," American Journal of Human Genetics, vol. 65, no. 1, pp. 50-58, 1999.

[16] Y. Dror, J. Donadieu, J. Koglmeier et al., "Draft consensus guidelines for diagnosis and treatment of ShwachmanDiamond syndrome," Annals of the New York Academy of Sciences, vol. 1242, pp. 40-55, 2011.

[17] S. A. Miller, D. D. Dykes, and H. F. Polesky, "A simple salting out procedure for extracting DNA from human nucleated cells," Nucleic Acids Research, vol. 16, no. 3, p. 1215, 1988. 
[18] A. Minelli, E. Nicolis, Z. Cannioto et al., "Incidence of Shwachman-Diamond syndrome," Pediatr Blood Cancer, vol. 59, no. 7, pp. 1334-1335, 2012. 

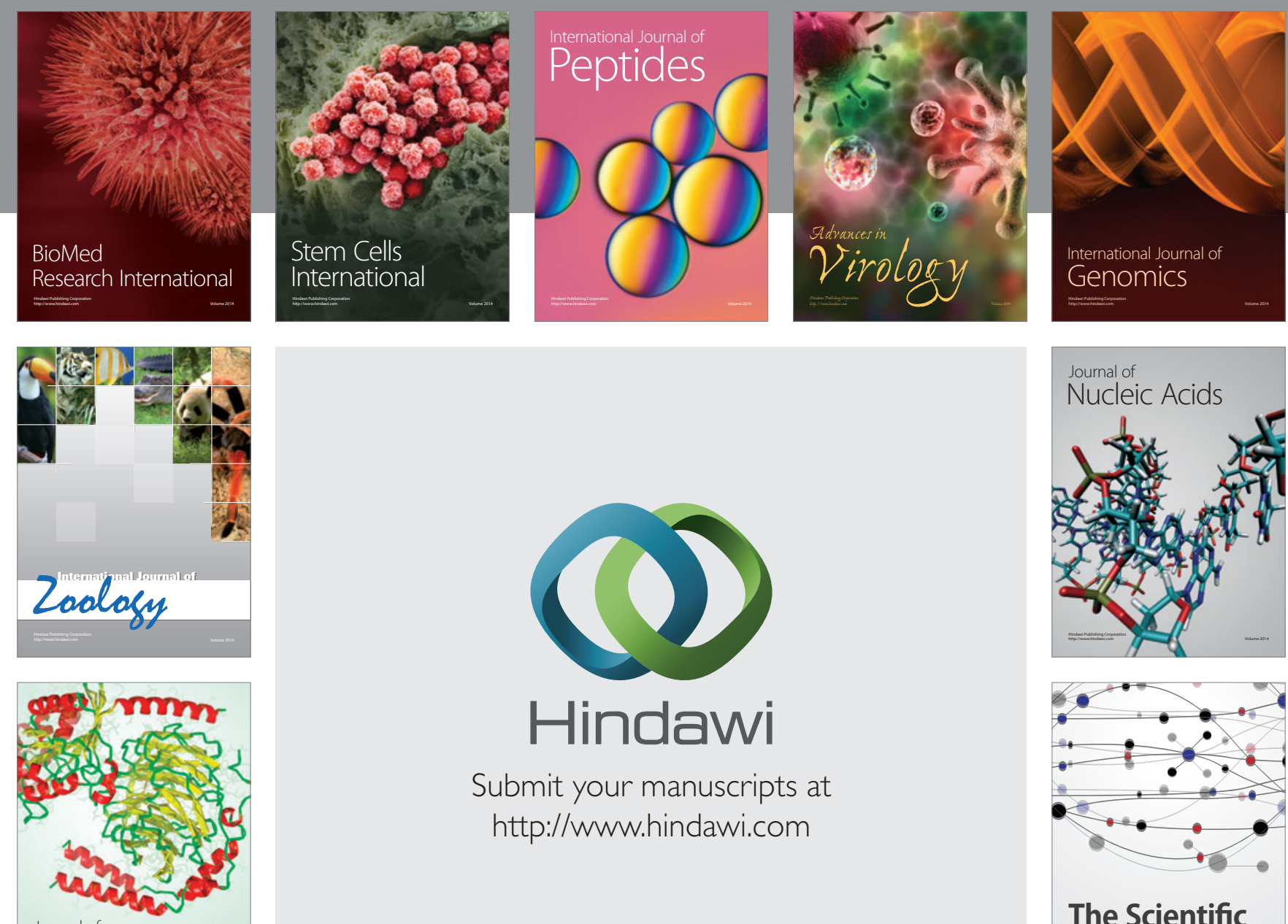

Submit your manuscripts at

http://www.hindawi.com

Journal of
Signal Transduction
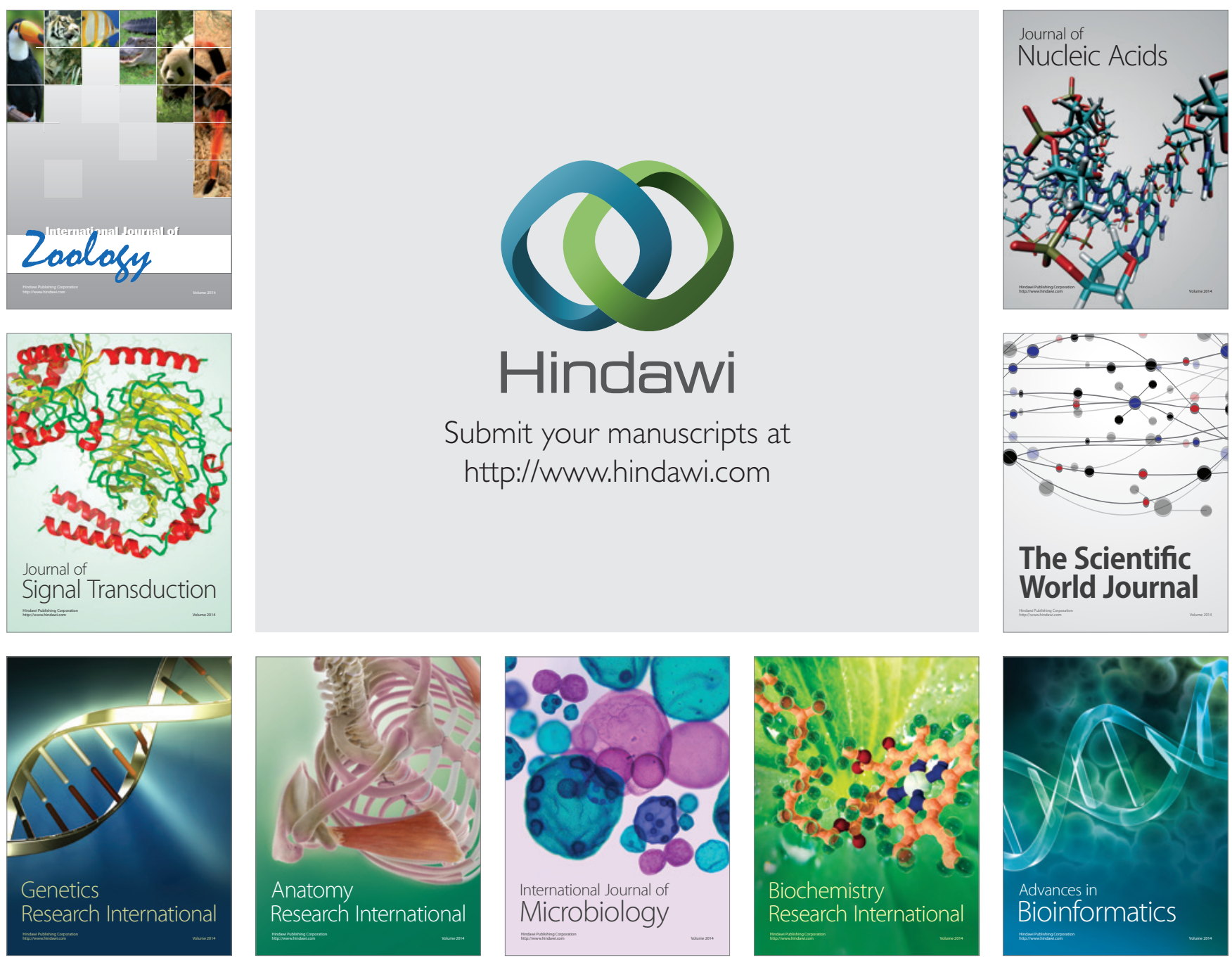

The Scientific World Journal
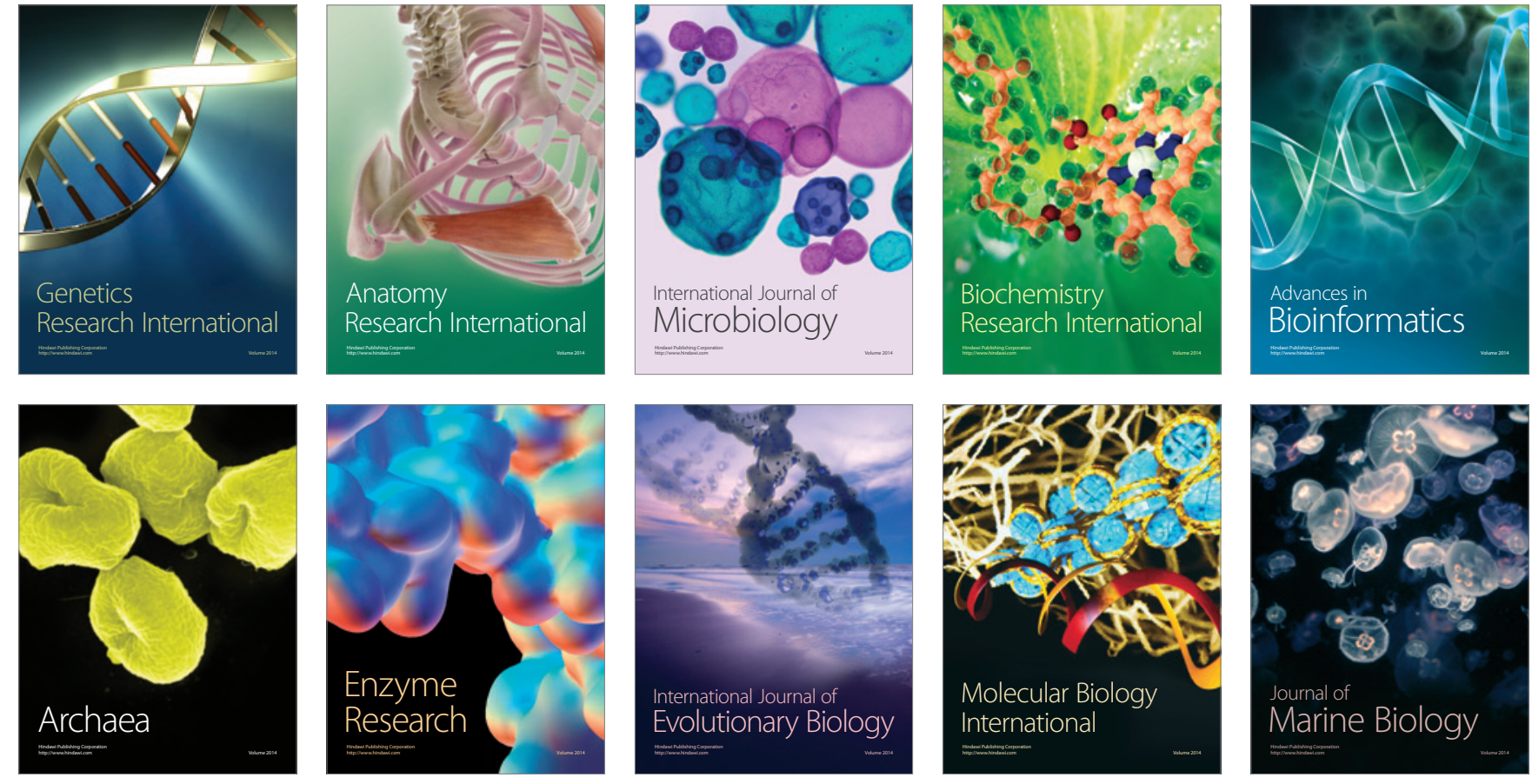